\title{
Development of Digital Image Processing using Fuzzy Gaussian Filter Tool for Diagnosis of Eye Infection
}

\author{
Jyoti Patil \\ Assistant Professor \\ I2IT,Hinjewadi, Pune-411057 India
}

\author{
A. L. Chaudhari \\ Phd, H.O.D. \\ Department of Electronics \\ MGSM's Arts, Science \& Commerce College, \\ Chopda Dist. Jalgaon 425107 India
}

\begin{abstract}
Diabetic retinopathy eye disease which is harmful, cuases pressure in eye nerve fiber. Therefore it is essential to diagnose it earlier. In this paper we have process on images of retina with the help of Digital

Processing (DIP) tool. In which images are getting detected and then processed. We distinguish the problem of detecting edges in images as a fuzzy logic problem. The edge detection problem can be separated into three stages: filtering, detection, and tracing. Images separated with the application of fuzzy reasoning based on local pixel characteristics which can control the degree of Gaussian smoothing. Filtered images are then applied to a simple edge detection algorithm which evaluates the edge fuzzy association value for each pixel, based on local image characteristics [1].
\end{abstract}

\section{Keywords}

Diabetic Retinopathy, Digital Image Processing, MATLAB, Fuzzy Gaussian Filter

\section{INTRODUCTION}

Eye is most important organ for human beings; it helps them sense the color, shape and state of physical objects. But eye infections like Fungal Keratitis, fungus aspergillus in the corneal layers is one of the major causes of blindness. In this work an attempt has been made to assist the diagnosis of Fungal Keratitis by identifying the region of infection in the corneal images using fractal-based features [2].

Diabetic retinopathy is the most common cause of blindness which a complication of diabetes mellitus. So to diagnose it early, the complicated images obtained from infected eye will be processed using DIP technique, which extract the information from the image. The Fuzzy Gaussian filtering algorithm is very immune to particular information content in an image. The Canny method finds edges by looking for local maxima of the gradient of I. The gradient is calculated using the derivative of a Gaussian filter. This method is therefore less likely than the others to be fooled by noise, and more likely to detect true weak edges.

In 2006 M.Hanmandlu, Rohan Raj Kalra, Vamsi Krishna Madasu, proposed a technique based on Univalue Segment Assimilating Nucleus (USAN) area i.e. fuzzy technique. The USAN characterizes the structure of the edge present in the neighborhood of a pixel and can thus be considered as a unique feature of the pixel and is fuzzified [3].In 2005 Zhang, Zhao and $\mathrm{Li} \mathrm{Su}$, proposed a technique based on the integer logarithm.Ratio of gray levels. In order to remove the ability of noise rejection they proposed a ratio of gray levels between the two successive image points rather than the difference [4] of gray levels to denote the variation in the gray levels Analyzing and interpreting retinal images have become a necessary and important diagnostic procedure in ophthalmology.

\section{METHODS OF DETECTION FUZZY GAUSSIAN FILTERS}

Edges are used for boundary estimation and segmentation in the scene. Since computer vision involves the identification and classification of objects in an image, edge detection is an essential tool. We characterize the problem of detecting edges in images as a fuzzy reasoning problem. The edge detection problem is divided into three stages: filtering, detection, and tracing. Images are filtered by applying fuzzy reasoning based on local pixel characteristics to control the degree of Gaussian smoothing. Filtered images are then subjected to a simple edge detection algorithm which evaluates the edge fuzzy membership value for each pixel, based on local image characteristics. Finally, pixels having high edge membership are traced and assembled into structures, again using fuzzy reasoning to guide the tracing process. The filtering, detection, and tracing algorithms are tested on several test images. Comparison is made with a standard edge detection technique [5].

Then edge detection is performed on the morphologically operated image. Laplacian and Gaussian operator detects the blood vessels accurately. Then thresholding is performed on the edge detected image. The blood vessel edges are thinned to a single line width. Then the blood vessels are smoothed. Smoothing function is used to smooth the thinned image for the betterment of blood vessel extraction. The smoothing is performed using box method with window size. Figure 1 shows Flowchart for Gaussian filter (Extracted blood vessels).[6] 


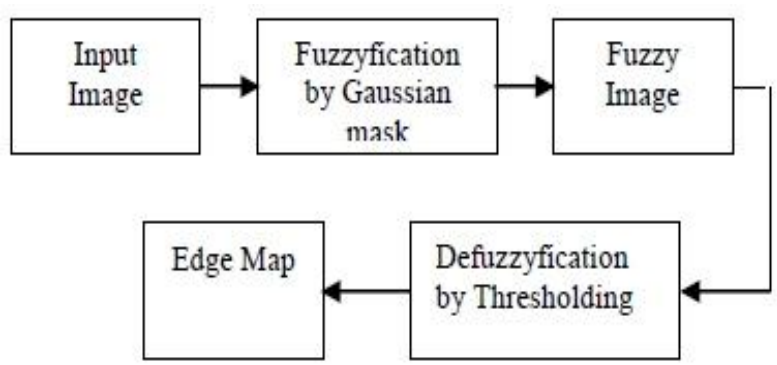

Fig. 1 Flowchart for Guassian filter (Extracted blood vessels)

\section{RESULT \& DISCUSSION}

The concept of Canny filter detection of signals is used to detect piecewise linear segments of blood vessels in these images. To identify the region of interest for blood vessel classification, it is essential to locate the optic disc initially. This algorithm of Gaussian filter is capable to detect looping structure in the blood vessel which is to manifestation of abnormal symptom. Thus to fabricate a complete retinal image analysis system that will assist \& improve the screening of diabetic retinopathy [7].

Fig 2 A) shows original diabetic image having red background. Fig 2 A) Original grayscale Image has a uniform grey background. So same grayscale image is enhanced

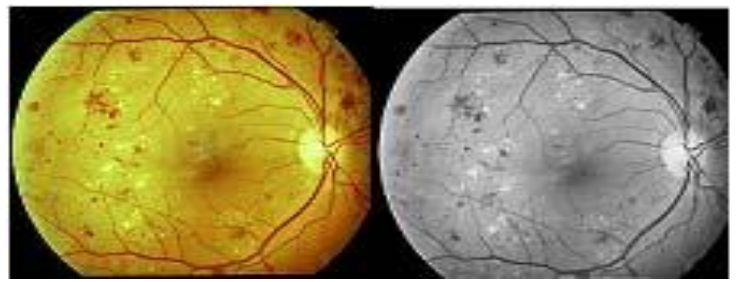

Fig.2(A) Original red background Image,(B) Original grayscale Image,

Fig (2)(C)\&(D) Shows the effect application of Edge etection algorithms of Canny edge detection with Mask 15x3) method on diabetic images using function of Canny Filter.

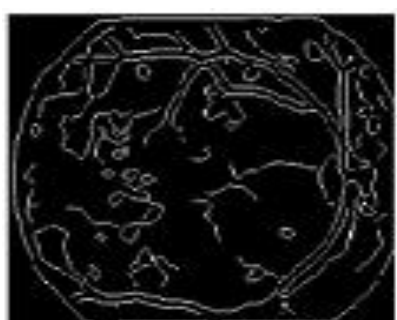

(C)

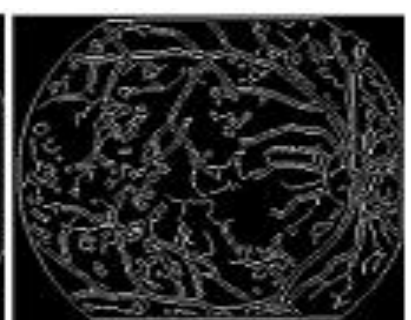

(D)
Fig 3 C) Canny edge,sigma $=1 \&$ D) Canny edge,sigma $=2$. Images after filtering

fig(3) (A) Is original grayscale Image in fig (3) (B) we can observe classified blood vessels, fig.(C) is Low-threshold image using Gaussian filter method.
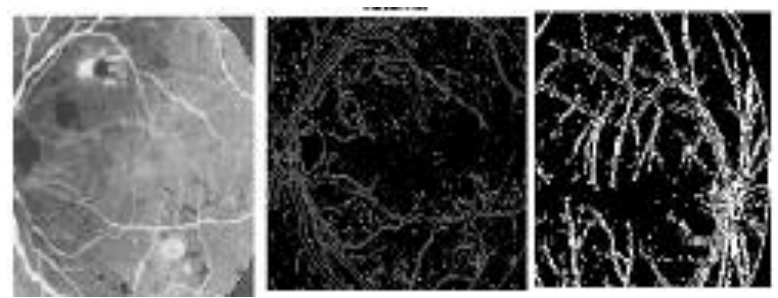

. Fig.3 (A) Original Image, (B) Classified vessals, (c) Low-threshold image In fig(4) we have extracted blood vessels. In fig (4)

(B)low threshold is applied (C) we can easily detect edges of blood vessels due to high threshold.

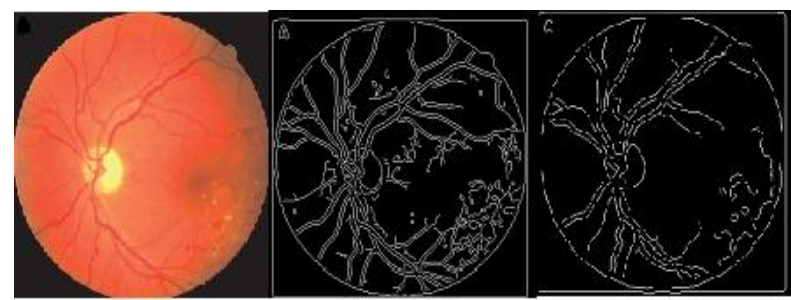

Fig.4 (A) Original Image,(B) low-threshold image,(C) highthreshold image Extracted blood vessel with Gaussian filter

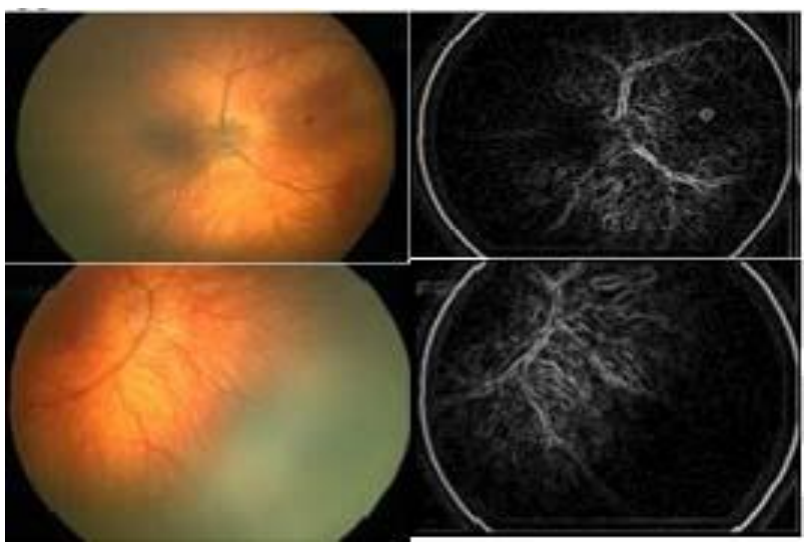

Fig 5. A) Original Image B) Images after Fuzzy Gaussian Filter Filtering

\section{CONCLUSION}

Present work uses Fuzzy Gaussian Filter feature extraction of images. It is very essential to extract infected blood vassals to diagnose early therefore filtering is frequently operated in image processing to smooth noise \& to enhance or detect features within an image. To improve quality of image linear \& non-linear method may be used [8].In future we are trying to develop an algorithm which can easily calculate the total area of infection also it can measure total diameter of related nerve fiber.

\section{ACKNOWLEDGMENTS}

I express my thanks to Dr. A.D.Shaligram, Head of Department of Electronics, University of Pune, for sharing his valuable knowledge. 


\section{REFERENCES}

[1] Bill Silver, "An Introduction to Digital Image Processing", Cognex Corporation, Modular Vision SystemsDivision,2000.

[2] "computer assisted eye fungal infection diagnosis", A Thesis Submitted to the Graduate Faculty of the Louisiana State University and Agriculture and Mechanical College in partial fulfillment of the requirements for the degree of Master of Science in Systems Science

[3] M. Hanmandlu, Rohan Raj Kalra, Vamsi Krishna Madasu,Shantaram Vasikarla."Area based Novel Approach for Fuzzy Edge Detection”. Department of Electrical Engineering, Indian Institute of Technology, Delhi.

[4] Ma, L., Tan, T., Wang, Y., Zhang, D.: "Personal Recognition Based on Iris Texture Analysis", IEEE Trans.Pattern Analysis and Machine Intelligence,25(12) (2003) 1519- 1533.

[5] Renyan Zhang, Guoling Zhao, Li Su. "New edge detection method in image processing". College of Autom., Harbin Engineering University, China.
[6] Big Fang,Wynne, HsuMong Li Lee"' On the Detection of Retinal Vessels in Fundus Images", Singapore-MIT Alliance, National University of Singapore, Department of Computer Science, School of Computing National University of Singapore.

[7] Iqbal, M.I , Aibinu, A.M ,Gubbal, N.S.Khan,“Automatic diagnosis of diabetic retinopathy using fundus images", Master's thesis Blekinge Institute of Technology October 2006

[8] Jing Wan, Xiaofu He, Pengfei Shi ," An Iris Image Quality Assessment Method Based on Laplacian of Gaussian Operation", Institute of Image Processing and Pattern Recognition, MVA2007 IAPR Conference on Machine Vision Applications, May 16-18, 2007, Tokyo, JAPAN.

[9] www.utdallas.edu/ dxa081000/IMAGEFILTERING. International conference proceedings, "Proceedings of the DRDO sponsored eighth Control Instrumentation System Conference CISCON-2011 (An International Conference) November 3-6,2011, Karnataka, Manipal University, Manipal,” (Development of Digital Image Processing tool for diagnosis of eye infection using MATLAB). 